ARTÍCULO ORIGINAL

\title{
Revista Médica Hondureña: Noventa años de aportes en parasitología
}

\author{
Revista Médica Hondureña: ninety years of contributions in parasitology
}

\author{
Rina G. Kaminsky
}

Maestría de Ciencias (MSC) en Parasitología; Instituto de Enfermedades Infecciosas y Parasitología Antonio Vidal; Asociación Hondureña de Parasitología; Tegucigalpa, Honduras.

RESUMEN. Antecedentes: Este artículo conmemora el 90 aniversario de la Revista Médica Hondureña. Objetivo: Registrar artículos sobre parasitosis, comentar algunas investigaciones y documentar hallazgos. Metodología: Se consultó la revista en la Biblioteca Virtual en Salud de Honduras (www.bvs.hn), separando artículos en parasitología de 1930 a 2019.Los trabajos libres presentados en Congresos Médicos Nacionales en temas de parasitología se identificaron de suplementos 2003-2019. Resultados: Se identificaron 234 publicaciones en parasitología en 12 temas, resaltando 73 artículos sobre malaria, 24 de insectos/ arácnidos, 23 en tratamiento, 14 en teniasis y cisticercosis, 12 sobre Enfermedad de Chagas y leishmaniasis, respectivamente, y otros en menor número. Se reconoció la falta de investigación parasitológica. De 624 trabajos libres, 90 (14\%) ocuparon temas parasitológicos, 40 en malaria y 17 en Enfermedad de Chagas. Casi ningún trabajo libre se completa a publicación. Discusión: Urgen publicaciones de investigaciones solidas en parasitología desarrolladas cuidadosamente, que fortalezcan el sector salud y provean productos adecuados con metas a reducir desigualdades. La Asociación Hondureña de Parasitología (AHPA), fundada el 23 de junio 2000, promueve el desarrollo de la parasitología en Honduras a través de la investigación científica y actividades de educación continua.

Palabras clave: Honduras, Investigación, Parasitología, Publicaciones.

Recibido: 3-2-2020 Aceptado para publicación 01-03-2020

Dirección para correspondencia: Rina G. de Kaminsky

Correo electrónico: camilaestela12@yahoo.com

Declaración de relaciones y actividades financieras y no financieras y conflictos de interés: ninguna.

DOI: https://doi.org/10.5377//rmh.v88i1.11601

\section{INTRODUCCIÓN}

En 2020 la Revista Médica Hondureña (RMH) cumple noventa años de publicación ininterrumpida, es decir, una de las revistas científicas locales de mayor longevidad. Se creó a partir de mayo 1930, meses después de fundarse la Asociación Médica Hondureña (2 de noviembre de 1929), siendo su primer director el Dr. Antonio Vidal Mayorga. ${ }^{1}$ El primer número dejó establecido en la Introducción que la Revista "...es el órgano de publicidad de la recién fundada Asociación Médica Hondureña, con el propósito que sea un medio potente de difusión científica, haciéndola llegar hasta el último rincón del país donde viva algún médico que pueda leerla...". Ofrecía publicar todo trabajo original y de difusión científica enviados por los socios y médicos hondureños fuera y dentro del territorio nacional. Prometía igualmente publicar artículos escritos por médicos centroamericanos y de otros países con condiciones patológicas similares a las de Honduras. En ese primer número se publicó el primer artículo sobre tema entomológico: "Zancudos Anofeles de Honduras" por el Dr. Vidal. Al presente la RMH cuenta con una versión impresa (ISSN 0375-1112), siendo indexada por primera vez en el IndexMedicus en 1966, en LILACS en el año 2000 y a partir de 2005 ofrece una versión electrónica (ISSN 19957068) de fácil acceso a través de la Biblioteca Virtual en Salud de Honduras (BVS HN) (www.bvs.hn). ${ }^{1}$ A partir del año 2003 al presente la Revista edita uno o dos suplementos anuales con el objeto de documentar contenidos de los Congresos Médicos Nacionales (COMENAC), y/o combinados con congresos de carácter internacional, o conmemorar eventos especiales como los aniversarios de la RMH.

En conmemoración de los 90 años de publicación ininterrumpida sobre temas médicos y científicos nacionales de interés, se procedió a revisar las publicaciones sobre temas en parasitología. Tal revisión tiene como objetivos identificar artículos sobre diferentes aspectos de parasitología y las infecciones causadas por parásitos en la casuística local, identificar parasitosis que por su frecuencia o rareza obligaron un artículo, resumir brevemente algunos que llamaron la atención de la autora y documentar los hallazgos en un artículo de base para futuras revisiones. No fue un objetivo medir la calidad de las publicaciones ni tampoco su impacto. 


\section{METODOLOGÍA}

Para recuperar la información buscada se recurrió a la BVS HN, identificando temas pertinentes al revisar el índice de cada número de la revista; sin embargo, no se contabilizó la totalidad de artículos publicados hasta la fecha. Las publicaciones en parasitología se clasificaron por temas y se guardaron en carpetas identificadas por nombre incluyendo una "miscelánea" con artículos únicos sobre un parásito determinado. Se revisó igualmente los suplementos 20032019 para separar los temas de parasitología que fueron presentados como trabajo libre (TL) en los COMENAC, habiendo contabilizado la totalidad de estos según numerados en los suplementos.

\section{RESULTADOS}

Se formaron 12 carpetas temáticas, dividendo el tiempo de publicación en decenios, desde 1930 hasta 2019, presentadas en el Cuadro No. 1; los TL recobrados de los suplementos están desglosados por temas similares en 9 carpetas en el Cuadro No. 2. La autoría de los artículos con pocas excepciones ha

Cuadro 1. Número de artículos por temas sobre parasitosis publicados en la Revista Médica Hondureña (decenios), 1930-2019, Honduras.

\begin{tabular}{|c|c|c|c|c|c|c|c|c|c|c|c|c|c|}
\hline Decenio & Malar & $\begin{array}{l}\text { Geohel } \\
\text { y otros }\end{array}$ & $\begin{array}{c}\text { Insec/ } \\
\text { Arac }\end{array}$ & Tratam & Ameb & $T / c$ & Chagas & Leish & Miscel & Angio & Toxo & $\begin{array}{c}\text { Api } \\
\text { intes }\end{array}$ & Total \\
\hline $1930-40$ & 44 & 3 & 4 & 10 & 10 & 0 & 1 & 0 & 3 & 0 & 0 & 0 & 75 \\
\hline $1941-50$ & 9 & 0 & 16 & 5 & 1 & 1 & 0 & 0 & $1^{* *}$ & 0 & 0 & 0 & 33 \\
\hline $1951-60$ & 0 & 4 & 0 & 3 & 1 & 1 & 1 & 0 & 1 & 0 & 1 & 0 & 12 \\
\hline $1961-70$ & 0 & 6 & 0 & 0 & 1 & 1 & 2 & 2 & 3 & 0 & 1 & 0 & 16 \\
\hline $1971-80$ & 0 & 5 & 1 & 1 & 4 & 0 & 1 & 1 & 0 & 1 & 0 & 0 & 14 \\
\hline $1981-90$ & 0 & 1 & 0 & 1 & 0 & 4 & 0 & 1 & 0 & 3 & 2 & 1 & 13 \\
\hline $1991-00$ & 6 & 5 & 1 & 1 & 1 & 3 & 5 & 3 & 1 & 0 & 0 & 1 & 27 \\
\hline 2001-10 & 9 & 4 & 1 & 1 & 0 & 1 & 1 & 4 & 1 & 1 & 0 & 1 & 24 \\
\hline 2011-19 & 5 & 6 & 1 & 1 & 1 & 3 & 1 & 1 & 1 & 0 & 0 & 0 & 20 \\
\hline Totales & 73 & 34 & 24 & 23 & 19 & 14 & 12 & 12 & 11 & 5 & 4 & 3 & 234 \\
\hline
\end{tabular}

Abreviaturas: Ameb= amebiasis, Angio= angiostrongilosis abdominal, Api intes= apicomplexa intestinales, Geohel y otros= geohelmintos y otros, Insec/arac= Insectos $/$ arácnidos, Leish=leishmaniasis, Malar= malaria, Miscel= miscelánea, $\mathrm{T} / \mathrm{c}=$ teniasis/cisticercosis, Toxo= toxoplasmosis, Tratam= tratamiento. * ${ }^{*}$ incluyeron publicaciones sobre ascariasis, uncinariasis y estrongiloidiasis. ${ }^{*}$ Se informa sobre parasito urinario.

Cuadro 2. Número total de trabajos libres y número de trabajos libres distribuidos por temas de parasitología, presentados en el Congreso Médico Nacional, suplementos Congreso Médico Nacional de la Revista Médica Hondureña 2003-2019.

\begin{tabular}{|c|c|c|c|c|c|c|c|c|c|c|c|}
\hline Año & $\begin{array}{l}\mathrm{TL} \\
\text { total }\end{array}$ & Malar & Chagas & Leish & $\begin{array}{l}\text { Aracl } \\
\text { insec }\end{array}$ & $\begin{array}{l}\text { Paras } \\
\text { Intes* }\end{array}$ & $\mathrm{T} / \mathrm{C}$ & Toxo & Angio & $\begin{array}{l}\text { Dermo } \\
\text { paras }\end{array}$ & Total \\
\hline \multicolumn{12}{|l|}{2003} \\
\hline 04 & 15 & 4 & 0 & 0 & 0 & 0 & 0 & 0 & 0 & 0 & 4 \\
\hline 05 & 26 & 5 & 1 & 0 & 0 & 0 & 2 & 0 & 0 & 0 & 8 \\
\hline 06 & 35 & 5 & 0 & 0 & 0 & 0 & 1 & 0 & 0 & 0 & 6 \\
\hline 07 & 29 & 2 & 2 & 1 & 2 & 0 & 0 & 0 & 0 & 1 & 8 \\
\hline 08 & 50 & 4 & 3 & 1 & 0 & 0 & 0 & 0 & 0 & 0 & 8 \\
\hline 09 & 42 & 3 & 0 & 1 & 1 & 0 & 0 & 1 & 1 & 0 & 7 \\
\hline 2010 & 20 & 2 & 2 & 0 & 0 & 0 & 0 & 0 & 0 & 0 & 4 \\
\hline 11 & 29 & 2 & 2 & 0 & 0 & 0 & 1 & 0 & 0 & 0 & 5 \\
\hline 12 & 29 & 0 & 2 & 0 & 0 & 0 & 1 & 0 & 0 & 0 & 3 \\
\hline 13 & 24 & 1 & 1 & 1 & 0 & 1 & 0 & 0 & 0 & 0 & 4 \\
\hline 14 & 30 & 4 & 1 & 0 & 0 & 3 & 0 & 0 & 1 & 0 & 9 \\
\hline 15 & 32 & 2 & 0 & 0 & 0 & 3 & 1 & 0 & 1 & 0 & 7 \\
\hline 16 & 27 & 1 & 0 & 0 & 0 & 1 & 0 & 0 & 0 & 0 & 2 \\
\hline $17^{\star *}$ & 57 & 1 & 3 & 0 & 0 & 0 & 0 & 0 & 0 & 0 & 4 \\
\hline 18 & 62 & 0 & 0 & 0 & 0 & 0 & 0 & 0 & 0 & 0 & 0 \\
\hline 19 & 117 & 2 & 0 & 1 & 5 & 0 & 0 & 2 & 1 & 0 & 11 \\
\hline Total (\%) & 624 & $40(6.0)$ & $17(2.0)$ & 5 & 8 & 8 & 6 & 3 & 4 & 1 & $90(14.0)$ \\
\hline
\end{tabular}

Abreviaturas: Arac/insec= arácnidos/insectos, Angio= Angiostrongylus costaricensis, Dermoparas= dermatoparasitosis, Enf inf= enfermedades infecciosas, Leish= leishmaniasis, Malar= malaria, Paras Intes= parásitos intestinales, T/c= teniasis/ cisticercosis (en su mayoría neurocisticercosis), TL= trabajo libre. *Incluye geohelmintos y Strongyloides stercoralis. **En 2017 se celebró en Tegucigalpa el XIII Congreso Centroamericano y del Caribe de Parasitología y Medicina Tropical. Los resúmenes se publicaron en el Suplemento 2, Vol. 85, pgs. 1-114 de la RMH, con un total de 84 trabajos libres de profesionales nacionales y extranjeros, con un total de 44 trabajos libres en parasitología. Esos no se incluyeron en este Cuadro 2. 
provenido de personal médico laborante de hospitales: Hospital San Felipe y Hospital y Hospital Escuela, Tegucigalpa.

Los artículos publicados inicialmente (1930-1960) carecían de un formato organizado y uniforme tal como se acostumbra actualmente. A excepción de algunos trabajos de Vidal, ninguno era resultado de investigaciones, sino más bien presentación de casos o experiencias personales. Con pocas excepciones, el autor era único, el contenido era relatado en primera persona, ninguno incluía un resumen, ni métodos de investigación, análisis estadístico o una presentación clínica ordenada; pocos incluían cuadros, gráficas o fotografías en los resultados, tampoco se presentaba una discusión de los hallazgos y en su mayoría no se listaba la literatura consultada, al extremo que en muchos tampoco se identificaba al autor y su afiliación. No obstante, el contenido es aún hoy de lectura interesante y sorprendente, ya que por aquellos años se carecía del auxilio de laboratorios bien equipados con personal adiestrado en el diagnóstico de estas enfermedades (que aún perdura) y de drogas apropiadas para el manejo de etiología parasitaria, en especial de las tres parasitosis sobresalientes entonces: paludismo, amebiasis y parasitosis intestinales. En 1956 siendo Director de la RMH el Dr. César Zúniga, ${ }^{2}$ expresó en un editorial que la Directiva de la Revista "...ha tomado muy en cuenta lo importante que es en toda publicación el acercarse lo más que se pueda a la perfección..." y ejecutó algunas modificaciones en cuanto a la numeración, incluyendo en ese número un artículo de Trejos $\mathrm{A}$ y Zeledón $\mathrm{R}$ de la sección de Tesis de Grado y Ensayos de la Universidad de Costa Rica sobre "Normas para la preparación de trabajos científicos" con el propósito de hacer los trabajos "más completos, más científicos y así menos criticables". A partir de esa fecha hasta la actualidad diferentes directores de la RMH se han encargado de modernizarla introduciendo mejoras tales como mejor identificación de la revista en páginas interiores, aceptación de trabajos de investigación aprobados por un comité de ética, un resumen en español y uno en inglés, análisis estadístico de los datos, revisión por pares, inclusión de consentimiento informado, apego a reglas internacionales de publicación y embelleciendo las portadas con cuadros de pintores hondureños. A partir del nuevo milenio, la autoría se combina con varios autores de diversas instituciones académicas y de salud pública, con estudiantes de pre- y postgrado y en pocos casos con instituciones extranjeras.

Se clasificó 234 publicaciones de parasitología en 12 temas. Del total de 73 artículos sobre malaria, 53 fueron publicados en los primeros 20 años, ninguno en los siguientes 40 años (1951-1990) y 20 entre 1991-2019 (Cuadro No. 1). Un registro similar se observa para publicaciones en temas de insectos/ arácnidos y tratamiento sobre enfermedades parasitarias; al menos un artículo sobre un tema en particular pero no más de tres se identificó en cada decenio; tomando como ejemplo 1981-1990, se identificó un artículo sobre tratamiento de parasitosis, uno sobre leishmaniasis, 2 sobre toxoplasmosis, uno de apicomplexa intestinales, 3 sobre angiostrongilosis abdominal y ninguno sobre paludismo, amebiasis, parasitismo intestinal, teniasis, cisticercosis ó insectos/arácnidos. Desde las primeras investigaciones en Honduras de apicomplexa intestinales (Cryptosporidium spp., Cyclospora cayetanensis y Cystoisospora belli) y a pesar de su importancia en diarreas infantiles y como causantes de enfermedad severa o muerte en individuos inmunocomprometidos, solamente se encontraron tres artículos sobre ese tema en los últimos 33 años. ${ }^{3}$

\section{Suplementos de la RMH}

En el Cuadro No. 2 se desglosan los trabajos libres (TL) presentados durante los diferentes COMENAC desde que la $\mathrm{RMH}$ los publica en suplementos a partir del año 2003. Como todos están numerados, fue fácil determinar el total de $624 \mathrm{TL}$, de los cuales 90 (14.0\%) fueron sobre temas en parasitología. Sobresalen las presentaciones orales 0 en cartel de Alger y col. sobre malaria (37 TL) y algunos sobre leishmaniasis y Enfermedad de Chagas. Alrededor de 7 de 37 TL sobre malaria ya fueron publicados; del resto de los 83 TL se desconoce si fueron publicados en otras revistas científicas locales 0 internacionales. Por lo tanto, no se hará comentario sobre sus contenidos, excepto para resaltar que para que la información presentada sea considerada adecuada científicamente es necesario completar la investigación o el informe de caso clínico con una publicación con revisión por pares.

\section{DISCUSIÓN}

Esta discusión se enfocara en comentarios a lecturas seleccionadas. La urgencia de controlar el paludismo se refleja en los artículos entre 1930-1940; en las bananeras de la costa norte, Deeks ${ }^{4}$ afirmaba que el $80 \%$ de las consultas era debido al paludismo, lo que obligaba a una distribución de pastillas de quinina y otros medicamentos a todos los dispensarios de la United Fruit Co. para tratar a una población trabajadora de 150,000 personas distribuida en 700 millas cuadradas de cultivos. La quinina, sin embargo, no destruía los gametocitos siendo necesario utilizar plasmoquina, que tenía cierto grado de toxicidad. El artículo se interrumpe en la página 44.

Una serie de siete artículos muy bien organizados sobre el control de la malaria, reproducidos en 1935 del folleto recibido de la Reforma de Lima, Perú, por el Dr. Carlos Enrique Paz Soldán se publicaron bajo el título de "La Peste Verde", afirmando que tal enfermedad en aquellos tiempos ocasionaba una pérdida anual al Perú de cinco millones de soles de oro.

La investigación de malaria en Honduras en 1944, estudio llevado a cabo por Vidal como jefe del Departamento de Laboratorio y Encargado de Estudios de Malariología del Servicio Cooperativo Interamericano de Salud Pública (SCISP), detallaba el clima y topografía del país, su geología, hidrografía, meteorología, registrando una población total de 1,107,859 habitantes y 47,223 habitantes en Tegucigalpa (censo 1940), ilustrada con cuadros sobre la altura, precipitación, defunciones por malaria por años por sitios y otros más. ${ }^{5}$ Su metodología de 
trabajo incluyó registro de índices esplénicos y examen de frote y gota gruesa de sangre coloreados por Giemsa en población seleccionada de las ciudades principales del país. Los mayores índices esplénicos fueron identificados en Amapala, Choluteca, San Lorenzo, Juticalpa y Comayagua (36.24-20.41) y los menores en la costa norte, Gracias y Yuscarán (3.52-1.58). De sus conclusiones se extrae que el parásito predominante era Plasmodium vivax, la mayor prevalencia del paludismo correspondió a los pueblos situados entre 1,000 a 3,000 pies de altura, el índice esplénico fue más alto en niños entre las edades de 7 a 12 años y la presencia de Anopheles albimanus varió inversamente a la de $A$. pseudopunctipennis.

Vidal en 1947 dio a conocer los resultados de administrar atebrina como terapia supresiva contra paludismo durante la construcción de $75 \mathrm{~km}$ de carretera entre Potrerillos y Pito Solo en 1942 entre 1,800 trabajadores. ${ }^{6}$ Aunque expresa que su investigación no reunió las condiciones requeridas para una experiencia verdaderamente científica ya que "los diagnósticos se hacían muy de prisa y el trabajo de laboratorio también fue extremadamente inadecuado, y aún después siempre hubo dudas respecto a su exactitud por la falta de técnicos académicos debidamente entrenados", vale la pena resumir los resultados extraordinarios obtenidos. No menos de 100-200 trabajadores visitaban diariamente el dispensario en Manacal solicitando tratamiento para una sintomatología clínica atribuida a paludismo y demostrado en el $51.7 \%$ de positividad de 1,421 frotes sanguíneos. Se distribuyó atebrina a todo trabajador dos días por semana durante el tiempo de los trabajos y los beneficios en salud fueron obviamente magníficos e increíbles: el índice palúdico inicial de $70.6 \%$ por cada 100 trabajadores medido por frote de sangre en noviembre y diciembre 1942 bajó a $24.9 \%$ en los primeros 3 meses de 1943 y al $5.9 \%$ a los 6 meses siguientes. Los trabajadores no presentaron reacción tóxica al tratamiento, su condición física para el trabajo fue excelente y la terapia se convirtió en un factor decisivo para completar el proyecto carretero.

Durante 3 años, 1944-46, se publicaron 15 trabajos sobre diferentes especies de mosquitos Anopheles, Aedes, culicinos y otros capturados en diferentes lugares del país y clasificados por Vidal, describiendo características de la larva y el adulto. Tres nuevas especies, $A$. aquasalis, $A$. neivai y $A$. bellator no vistas anteriormente fueron descritas por Vidal, quien resume que entre identificaciones realizadas por otros investigadores antes y después de 1930 y las suyas en 1930 fija 16 especies ya identificadas en Honduras. ${ }^{7}$ Resaltaron como más importantes por su relación con Plasmodium vivax o P. falciparum: $A$. pseudopunctipennis abundante en estación seca, $A$. albimanus abundante en época de lluvia y probablemente el vector principal en Honduras y $A$. argyritarsis. Las especies más peligrosas fueron $A$. darlingi y $A$. punctimacula. Otras publicaciones entomológicas han incluido: una sobre intoxicación por Lactrodectus, una acerca de "Higiene rural. Moscas" sin autor (1933), miasis orbitaria, aracnidismo, escabiosis y otra sobre pediculosis, pero hasta la fecha no se registraron estudios adicionales en anofelinos. . $^{8-12}$

Desde finales de los 90 hasta el presente, los 15 trabajos publicados en la RMH por Alger y colaboradores en el tema de malaria sobre aspectos clínicos, casos graves y complicados, dengue y malaria, malaria subclínica, manejo, vigilancia, diagnóstico, etc., son de lectura obligada para todos aquellos estudiosos del tema. Alger posee un doctorado en Parasitología, es una científica cuidadosa en la investigación, de prosa precisa, con explicaciones claras, científicamente correctas y objetivas en las conclusiones. Destaco uno de su coautoría: "Malaria y embarazo"13 que sugiero contrastar con "Malaria en el embarazo" de Villar. ${ }^{14} \mathrm{El}$ propósito del estudio ${ }^{13}$ era determinar el impacto clínico de la malaria en la embarazada y su producto y por limitantes de espacio se menciona aquí apenas un destaque. En $1942^{14}$ el autor afirmaba que los textos de obstetricia comentaban de la poca influencia de malaria en el embarazo y apenas un factor en abortos y partos prematuros, con muchas dudas y vacilaciones, especialmente en el tratamiento, pues existía la impresión que la quinina podía causar abortos. Fernández y col. por el contrario, demostraron los estragos de esta parasitosis en la mujer embarazada y su producto. Las complicaciones más frecuentes en 34 embarazadas del Hospital Atlántida en La Ceiba fueron amenaza de parto prematuro y parto prematuro. Todos los casos se presentaron como infecciones febriles agudas; en la época del estudio (1981-82) la Región Sanitaria 4 aportaba casi la mitad de todos los casos nacionales, siendo la intensa exposición al parásito el factor más influyente en la presentación clínica. El antecedente de malaria fue significativo en más casos que en controles; la anemia moderada o severa ( $9-<7 \mathrm{~g} / \mathrm{dL}$ de hemoglobina) fue asociada significativamente a malaria $(p=0.0001)$ y uno de los productos tuvo malaria neonatal (Plasmodium falciparum). Dos mujeres abortaron y 4 productos fueron prematuros, 2 fallecieron (causa desconocida por parto en domicilio). De las 14 embarazadas y 5 puérperas del Bloque Materno-Infantil del Hospital Escuela (HE) con malaria, en el $60 \%$ el diagnóstico fue incidental, el $73.6 \%$ residía en área endémica; hubo 4 transmisiones congénitas en embarazadas con infección subclínica, una muerte materna y 3 muertes neonatales. Los síntomas clínicos (HE) fueron cuadro febril agudo acompañado de complicaciones como disuria, vaginitis, actividad uterina (26.7\%), anemia con o sin ictericia (46.7\%). La cloroquina fue el medicamento más seguro y de buena respuesta terapéutica. Otro artículo reciente muy detallado discute la importación de casos de malaria de África en donde predomina $P$. falciparum resistente a la cloroquina y la necesidad nacional de una vigilancia de calidad..$^{15}$

Exceptuando el decenio $1930-40$ y el 1971-80 con 10 y 4 publicaciones respectivamente, se contabilizaron 5 artículos adicionales de 1981 a 2019 para un total de19 publicaciones sobre amebiasis, notando artículos sobre absceso hepático amebiano en 1936 y como tema oficial de Honduras con 61 casos recolectados durante cinco años y medio presentado en 
el congreso médico centroamericano celebrado en El Salvador en $1964 .{ }^{16}$ Impresiona el tratamiento de la disentería amebiana entonces: inyecciones de emetina, 12 o más, combinado con lavados con Yatrén al $1 \%$ ó 2\%, que se repiten lo más posible durante toda la noche y por varios días; yoduro doble de bismuto, jarabe de ipeca, arsenicales. ${ }^{17}$ Paredes en su publicación "EI petróleo en las colitis amibianas y tricomonas" describe utilizar lavados de petróleo o kerosene, gas usado en candiles o quinqués, en por lo menos 80 casos tratados en el Hospital General y La Policlínica. ${ }^{18}$ La cantidad de líquido variaba a lo que el paciente pudiera soportar con retención de entre media hora a 36 horas; tratamiento incluso administrado a sus hijos que padecían de amebiasis desde la edad de un año, y a sí mismo, con un costo mínimo de 18 centavos la botella, con total y pronta curación y sin efectos secundarios (!). A finales del siglo pasado (1993) el conocimiento científico relacionado a Entamoeba histolytica patógena tuvo un giro importante con la separación de amebas no patógenas morfológicamente idénticas por medio de pruebas moleculares, genéticas, histoquímicas, inmunológicas, etc. y que aún no termina, ${ }^{19}$ por lo que suponemos que la amebiasis en aquellos años (y aún en el presente) era sobrediagnosticada y la verdadera etiología de la enfermedad se vio privada de un diagnóstico y tratamiento apropiados.

Tal vez la primera investigación en el tema de parasitismo intestinal en Honduras fuera realizada en escuelas de Tegucigalpa en 1941 (Zepeda J. Los parásitos intestinales en las escuelas primarias del Distrito Central. Revista Honduras Farmacéutica 1941; 7), seguido en los años 70 por otras encuestas en escolares del distrito central y Choluteca. ${ }^{20,21}$ Hoekenga(1950) publicó resultados de examen de una muestra de heces en 5,000 personas en la costa norte en una revista científica extranjera. ${ }^{22}$ Esos y otros estudios subsiguientes arrojaron altos porcentajes de parasitismo intestinal por helmintos $(>80 \%)$ y protozoos $(>30 \%)$, dato que ha variado poco en los últimos 60 años. Gutierrez López documentó en 1954 seis casos de perforación intestinal por parásitos en 5 pacientes adultos y una adolescente que requirieron cirugía, habiendo fallecido todos. ${ }^{23}$ Los hallazgos quirúrgicos informaron 3 úlceras amebianas perforadas, masa en íleo terminal, invaginación y perforación a la pared intestinal encontrando una tenia completa, perforación en íleo distal y Ascaris lumbricoides, masa necrótica en parte distal de íleo, intususcepción y perforación, recobrando ovillo de gusanos adultos de Trichuris trichiura. Dice conocer de otros casos similares que no describe por carecer de datos completos y con gran desaliento afirma que "el problema de parasitismo intestinal en nuestro país es insoluble". Dos muertes adicionales, una por infección masiva de T. trichiura en un niño luego de 5 meses de hospitalización y múltiples tratamientos, ninguno específico contra tricuriasis y otro por perforación de colon en una balantidiasis en un adulto de 24 años de edad fueron ilustrados con fotografías. . $^{24,25}$ Ahora sabemos que los programas de quimioterapia preventiva promovidos por la Organización Mundial de la Salud no solo son exitosos en controlar las helmintiasis sino que tienen importantes beneficios colaterales ${ }^{26}$ Estudios de morbilidad y/o mortalidad por geohelmintos y protozoos en Honduras es un tema huérfano de interés.

Cuando Hoekenga publicó el artículo "Toxoplasmosis en Honduras" (1956) se desconocía totalmente el ciclo de vida del agente causal Toxoplasma gondii y apenas en 1939 se le había imputado como importante causa de corioretinitis focal, hidrocefalia, convulsiones, encefalomielitis y enfermedad congénita entre otras. ${ }^{27}$ Hoekenga utilizó una prueba tintoreal (Sabin-Feldman) en 266 pacientes divididos en 7 grupos etarios, correlacionó los títulos con la procedencia geográfica y los títulos más altos con alguna clínica, además de estudiarla en perros y cerdos. La prevalencia de positivos fue de $89 \%$ en mujeres y $95 \%$ en hombres de muchos lugares de Honduras como Intibucá, Comayagua, Santa Bárbara, La Ceiba y muy diseminada en el Valle del Ulúa. De los 170 positivos (63.9\%), ocho presentaron títulos muy altos (1:1,0241:4,096), seis niños entre 5 y 10 años, un joven de 19 años y un adulto de 40 años. Lo común entre todos fue fiebre de origen indeterminado y conjuntivitis o querato-conjuntivis. Se encontró que los perros estudiados tenían $86 \%$ de positividad. Más tarde (1969), ${ }^{28}$ Cardona publicó los dos primeros casos congénitos y Grinspan, Henriquez y Madrid (1988) ${ }^{29}$ describieron otro caso congénito con síndrome de Torch, habiendo encontrado múltiples calcificaciones y pseudoquistes cerebrales en el postmortem del paciente de 37 días de nacido; estos autores incluyeron una figura mostrando el recién reconocido (1970) ciclo de vida del parásito. A excepción de un trabajo libre en embarazadas presentado como conferencia [Rivera M. 25C. Detección precoz de toxoplasmosis congénita. LII Congreso Med Nac; Rev MedHondur 2009; 77 (Supl No.1):S27], no se conocen investigaciones sobre toxoplasmosis en Honduras publicadas en la RMH.

Los primeros siete casos de cisticercosis dados a conocer por Cueva (1956) ${ }^{30}$ fueron seguidos de otros 22 casos, producto de una revisión de 35,000 biopsias en 15 años (1951-1966) por Durón, ${ }^{31}$ que representaban únicamente aquellos que tuvieron estudios histológicos por el único Departamento de Patología a nivel nacional en el Hospital General San Felipe. La localización de los cisticercos varió entre tejido subcutáneo, muscular, pulmonar, ojo y anexos y 7 en sistema nervioso central, algunos pacientes con cisticercos en múltiples localizaciones. Durón incluyó datos de prevalencia de teniasis en un total no especificado de exámenes de heces durante seis años (1960-66) en el Hospital San Felipe, de 112 casos por año. Desde entonces a la fecha se publicaron dos revisiones sobre neurocisticercosis: una (1986) de 20 casos en niños, ${ }^{32}$ el 100\% diagnosticado por radiografía de cráneo en el HE y la segunda (1989) de 129 casos por revisión retrospectiva de expedientes del HE, notando que la oferta de tomografía axial computarizada y una prueba específica de inmunoabsorción ligada a enzimas (ELISA) (ofertada privadamente) cuadruplicaron la posibilidad del diagnóstico, de 26 casos diagnosticados en 5 años (198084), a 103 casos diagnosticados en 4 años (1985-88). ${ }^{33}$ Honduras (y en general a nivel mundial) tiene muchos vacíos de 
conocimiento acerca de la situación epidemiológica, que es clave para poder decidir en donde aplicar intervenciones. También hay dificultades con los métodos de diagnóstico individual y de diagnóstico en salud pública de teniasis humana y cisticercosis humana y animal, vitales para la conducción de un programa de control eficaz de esta zoonosis transmitida por alimentos más importante a nivel mundial (http://iris.paho.org). ${ }^{34}$ Otros informes de céstodos reconocidos en el país incluyen Raillietina quitensis, hidatidosis cerebral, sacra y de tejidos blandos y huevos de pseudofilideo en perro. ${ }^{35-39}$

En Honduras sucedió algo similar a lo acontecido en Brasil con la Enfermedad de Chagas: el parásito fue descrito en frotes de sangre de una zarigüeya capturada en Tela $(1931)^{40}$ antes de describir los primeros casos clínicos en $1960 .{ }^{41}$ Dala Sierra en 1995 reprodujo el artículo original y aportó valiosos comentarios al respecto. ${ }^{42}$ La primera demostración histológica del parásito (1965) y la primera descripción del signo de Romaña y su importancia semiológica (1971) (3,44 $^{4}$ fueron preámbulos necesarios a la primera encuesta serológica y parasitológica en 15 departamentos de Honduras en 1983-1986, cuyos resultados fueron dados a conocer en la Serie de Diagnósticos No. 6 de la Organización Panamericana de la Salud en 1992. La investigación ininterrumpida de campo sobre Trypanosoma cruzi y sus dos vectores importantes en Honduras por Carlos Ponce y col. abarcó más de 30 años y dio origen a docenas de publicaciones en revistas extranjeras, lo que dejó huérfana a la RMH y a sus lectores en lengua español exclusiva de esa riqueza en conocimientos pioneros en el país. Sin embargo, los resultados lograron demostrar que la Enfermedad de Chagas era un serio problema de salud pública local. Los logros obtenidos incluyen la notificación obligatoria de la Enfermedad de Chagas en Honduras, la certificación de haber eliminado la transmisión aguda de T. cruzi por Rhodnius prolixus en noviembre 2010; el control de la transmisión transfusional en los Bancos de Sangre nacionales que ha sido exitoso, la instauración de un programa de colocación de marcapasos, un manejo adecuado de casos agudos, además de fortalecer el diagnóstico y tratamiento de casos congénitos.

A partir de 1967 y hasta la fecha las publicaciones sobre leishmaniasis se reducen a 11 artículos y una base de datos, describiéndose 100 casos de leishmaniasis en el primer artículo, 67\% cutánea, 32\% muco-cutánea y un caso de leishmaniasis diseminada ${ }^{45}$ Varias publicaciones sobre revisión de expedientes del Hospital Escuela pusieron en relevancia la severidad de leishmaniasis visceral en población infantil, cuyo primer caso fue publicado en 1974 en una niña de 16 meses de edad que falleció, habiendo comprobado las formas intracelulares del parásito en histiocitos de médula ósea y en tejido hepático y bazo. ${ }^{46-48}$ Honduras fue la primera en informar en la literatura mundial sobre otra presentación clínica causada por Leishmania chagasi, la leishmaniasis cutánea no ulcerada (LCNU) o leishmaniasis cutánea atípica (LCA) ${ }^{49}$ Esta especie causa leishmaniasis visceral, que es mortal si no se trata a tiempo; sin embargo, en casos provenientes del sur del país se la diagnostica como LCNU en individuos jóvenes, sanos y sin progresión a úlceras cutáneas ni visceralización. ${ }^{50} \mathrm{La}$ investigación robusta de tal peculiaridad inusual está en espera y ojalá los resultados sean dados a conocer en la RMH. Una recopilación de casos clínicos y características epidemiológicas de pacientes atendidos durante dos años en el Hospital Escuela con seguimiento clínico y terapéutico representa un informe ejemplar de la leishmaniasis en Honduras. ${ }^{51}$

\section{COLOFÓN}

Interés en la enseñanza de parasitología tanto en precomo en postgrado en la Facultad de Ciencias Médicas UNAH o como especialidad en el área de la salud en Honduras es débil 0 inexistente. El desinterés por incluir esta materia de manera formal en el currículo educativo con profesionales especializados, acompañada de laboratorios de práctica y de investigación, desasocia la mejora del conocimiento médico en lo referente a enfermedades parasitarias crónicas y al reconocimiento de pacientes en riesgo, limitando severamente el interés en la promoción de investigaciones afines. Programas organizados que atiendan estas necesidades son urgentes para proveer de conocimiento afín a los futuros profesionales y disminuir disparidades en poblaciones vulnerables. La "pausicidad" de investigaciones en esta área de la ciencia, coloca la medicina del país en desventaja regional.

Una revisión reciente de trabajos libres durante 11 años concluyó que, aunque solo aquellos trabajos de mayor calidad y rigor científico son presentados en los COMENAC, ello no se asociaba con mayor índice de publicaciones posteriores. ${ }^{52}$ Recomendaban los autores incentivar la investigación desde el pregrado y procurar medios necesarios al proceso, para así transferir los resultados en publicaciones utilizables por otros autores. Para la mejora continua de la RMH se estipula "...que la publicación es la culminación de un ciclo de investigación en el cual se debe contar con un sistema institucional y nacional de investigación para la salud fortalecido, con gobernanza sólida, con marco legal y regulatorio inclusivo, que responda a principios y valores éticos, con priorización de la investigación innovadora de acuerdo a las necesidades de la población y acceso a recursos financieros que promuevan el desarrollo continuo científico y tecnológico. La falta de investigaciones éticas y de calidad limitan directamente la disponibilidad de artículos originales y de manera indirecta la disponibilidad de todos los otros tipos de artículos que publica la Revista". ${ }^{53} \mathrm{El}$ futuro, sin embargo, debe considerarse alentador, afirmando un estudio reciente que la investigación científica y la producción en Honduras está mejorando con mayor número de artículos y citas en SCI-EXPANDED, lo cual se traducirá en beneficios para la población hondureña. ${ }^{54}$

Hace ahora 20 años, precisamente el 23 de junio de 2000 se fundó la Asociación Hondureña de Parasitología, AHPA por 
sus siglas, cuyo objetivo se definió como promover el desarrollo de la parasitología en Honduras y proporcionar la superación académica y cultural de sus miembros. Cumpliendo tal objetivo, miembros de la AHPA han participado con presentación de trabajos científicos originales en congresos científicos médicos y de parasitología, nacionales e internacionales, incluyendo congresos bianuales de la Federación Latinoamericana de Parasitología (FLAP), dos congresos de la Federación Mundial de Parasitólogos (ICOPA) y con la organización local de dos congresos internacionales de parasitología y medicina tropical (2001 y 2017) de la Asociación Centroamericana y del Caribe de
Parasitología y Medicina Tropical (ACCAPMT). ${ }^{55}$ Su actividad más destacada ha sido un análisis de la calidad de diagnóstico parasitológico en laboratorios de Tegucigalpa ${ }^{56}$ previo al diseño e implementación de cursos de educación continua sobre el diagnóstico parasitológico laboratorial. ${ }^{57}$ Con responsabilidad social y con mucha dificultad se está contribuyendo a mejorar el desempeño de profesionales en el laboratorio y a la promoción de la investigación sobre preguntas importantes en parasitología, cuyos resultados son indispensables como evidencia científica al considerar acciones en el manejo de los problemas parasitológicos en la población.

\section{REFERENCIAS}

1. Javier Zepeda C. Reseña histórica de la Revista Médica Hondureña 19302005. Rev Med Hondur. 2005; 73(Supl 2):4-16.

2. Zúniga C. Página del Director. Rev Med Hondur. 1956; 24:1-2.

3. Kaminsky RG. Comparación clínica y epidemiológica entre apicomplexa intestinales en población hospitalaria en Honduras. Rev Med Hondur. 2002; 70(4):164-162.

4. Deeks WE. Medidas para controlar el paludismo. Rev Med Hondur. 1930; A0(2):39-44

5. Vidal A. Investigación de malaria en Honduras. Rev Med Hondur.1944; A14(4):487-502.

6. Vidal A. Terapia supresiva del paludismo por medio de la atebrina. Rev Med Hondur. 1947; A17(2):405-410.

7. Vidal A. Zancudos anofeles de Honduras. Rev Med Hondur. 1944; A14(3):439-458.

8. Bulnes M. Intoxicación por un arácnido venenoso, la casampulga (Lactrodectus nactus o nactans). Rev Med Hondur. 1943; A13(2):114-116.

9. Elvir Aceituno C. Miasis orbitaria por Dermatobia hominis. Rev Med Hondur. 1974; 42:255-257.

10. Aronne G. Aracnidismo: Lactrodectismo en Honduras. Rev Med Hondur. 1992; 60(1):39-41.

11. Morales S, Matute N. Lo que el médico general debe saber sobre escabiosis. Rev Med Hondur. 2008; 76:121-127.

12. Figueroa J, Moncada V, Reyes O, Peña C, Kaminsky RG. Pediculosis capitis: un problema de salud desatendido en Honduras. Rev Med Hondur. 2012; 80:102-106.

13. Fernández RD, García Y, Alger J. Malaria y embarazo: observaciones clínico-epidemiológicas en dos zonas geográficas de Honduras. Rev Med Hondur. 2001; 69:8-18

14. Villar RA. Malaria en el embarazo. Rev Med Hondur. 1942; A12(5):260263.

15. Nieto Zelaya VG, Alvarado Claros VG, García J, Alger J, Tovar Calderón S, Navarro JJ. Escolar con malaria por Plasmodium falciparum de África: riesgo para la salud pública de Honduras. Rev Med Hondur. 2019; 87:20-26.

16. Zuñiga SR, Alonso EM, Rivera JM, Rivera h J, Durón R, Lozano R. Absceso hepático amebiano. Rev Med Hondur. 1964; 32:22-43.

17. Larios Córdoba M. Amebiasis. Observaciones sobre la incidencia y el diagnóstico de la infección amebiana en Tegucigalpa. Rev Med Hondur.1930; A0(5):3-12).

18. Paredes $\mathrm{S}$. El petróleo en las colitis amibianas y tricomonas. Rev Med Hondur. 1943; $A 13(3): 151-154$.

19. Kantor M, Abrantes A, Estevez A, Schiller A, Torrent J, Gascon J, et al. Entamoeba histolytica: Updates in clinical manifestation, pathogenesis, and vaccine development. Can J Gastroenterol Hepatol. 2018; 2018:4601420.

20. Zepeda J. Estudio parasitoscópico en tres escuelas primarias de la zona sub-urbana de Tegucigalpa, Honduras. Rev Med Hondur. 1972; 40:119122.

21. Zepeda J, Barahora G. Estudio parasitoscópico escolar en Monjarás, Departamento de Choluteca, Honduras. Rev Med Hondur. 1971; 39:306309.

22. Hoekenga MT. The incidence of human intestinal parasites in northwestern
Honduras. Am J Trop Med Hyg. 1950; 30(5):757-759.

23. Gutierrez López R. Perforaciones intestinales por parásitos. Rev Med Hondur. 1954; 23(1):805-808.

24. Durón R. Conferencia clínico-patológica. Rev Med Hondur. 1968; 36:265269.

25. Durón R. Balantidiasis con perforación de cólon. Rev Med Hondur 1969; 37(4):294-300.

26. Hotez PJ, Fenwick A, Molyneux DH. Collateral benefits of preventive chemotherapy - expanding the war on Neglected Tropical Diseases. N E J Med. 2019; 380:2389-2391.

27. Hoekenga MT. Toxoplasmosis en Honduras. Rev Med Hondur. 1956; 23(175):160-165

28. Cardona V. Toxoplasmosis congénita. Rev Med Hondur. 1969; 37:301-310.

29. Grinspan S, Henriquez O, Madrid R. Toxoplasmosis congénita. Rev Med Hondur. 1988; 56: 279-283.

30. Cueva A. Primeros casos de cisticercosis en Honduras. Rev Med Hondur. 1956; 24:101-111.

31. Duron R. Cisticercosis en Honduras. Rev Med Hondur. 1967; 35:126-133

32. Cuellar R, Wood de Bush J. Cisticercosis cerebral en niños Hospital Materno-Infantil. Rev Med Hondur. 1986; 54:126-131.

33. Alvarez A, Nazar N. Neurocisticercosis en el Hospital-Escuela. Rev Med Hondur. 1989; 57:246-260

34. Organización Panamericana de la Salud, Organización Mundial de la Salud. Pautas Operativas para las Actividades de Control de la Teniasis y la Cisticercosis causadas por Taenia solium. Washington D.C.: OPS/OMS; 2019.

35. Vidal A. Raillietina quitensis L A. León. 1935. Una tenia encontrada por primera vez en Honduras. Rev Med Hondur. 1947; A17(4):504.

36. Cardona López V. Hidatidosis cerebral. Reporte del primer caso de hidatidosis en Centro América. Rev Med Hondur. 1966; 34:115-124.

37. Ferrera Erazo C, Henríquez O. Hidatidosis en tejidos blandos. Rev Med Hondur. 1989; 57:133-137.

38. Gonzáles Rull M, Cortés Moreno A, Valenzuela R. Hidatidosis sacra. Rev Med Hondur. 1981; 49:22-27.

39. Kaminsky RG, Groothousen C. Huevos de cestodo pseudofilideo en perros, Tatumbla, Honduras. Rev Med Hondur. 2014; 82:33-34.

40. Robertson A. Nota sobre un trypanosoma morfológicamente semejante al Trypanosoma cruzi (Chagas, 1909) encontrado en una zarigüeya (Didelphis marsupialis), capturado en Tela, Honduras. Rev Med Hondur. 1931; A2(14):3-13.

41. León Gómez A, Flores Fiallos A, Reyes Quesada L, Poujol ER, Bonilla MR, Gómez Padilla C. La Enfermedad de Chagas en Honduras. Rev Med Hondur. 1960; 28(2):43-49.

42. Dala Sierra E. Primera descripción de Trypanosoma en Honduras. Rev Med Hondur. 1995; 63:39-43.

43. Durón R. Miocarditis chagasica. Su primera demostración histológica. Rev Med Hondur. 1965; 33:240-249.

44. Laínez Nuñez H, Fernández Selva E. Forma aguda de la Enfermedad de Chagas, importancia semiológica del signo de Romaña. Rev Med Hondur. 1971; 39:5-15. 
45. Fernández E. Leishmaniasis en Honduras. Rev Med Hondur. 1967; 35:8388.

46. Nuernberger SP, Ramos CV. Leishmaniasis visceral. Informe del primer caso en Honduras. Rev Med Hondur. 1974; 42:234-241.

47. Dala Sierra E, Velásquez García O, Matamoros F. Leishmaniasis visceral en lactantes. Análisis de 29 casos. Rev Med Hondur. 1989; 57:4-13.

48. López A, Molina C, Bueso A, Fuentes F. Leishmaniasis visceral en niños. La experiencia en 35 casos. Rev Med Hondur. 1991; 59:123-129.

49. Ponce C, Ponce E, Morrison A, Cruz A, Neva F. Leishmania donovani chagasi: new clinical variant of cutaneous leishmaniasis in Honduras. Lancet 1991; 357:67-70.

50. Lizardo Castro G, Ponce C, Ponce E, Sierra M. Caracterización de pacientes con leishmaniasis cutánea atípica. Rev Med Hondur. 2008; 76:101-107.

51. Matute N, Espinoza C, Alger A, Padgett D, López E, Zúniga C. Caracterización clínico-epidemiológica de pacientes con leishmaniasis atendidos en el Hospital Escuela. Rev Med Hondur. 2009; 77:7-15.

52. Castejón Cruz OA, Núñez Savoff RA, Bush Wood SW. Características de los trabajos de investigación presentados en los Congresos Médicos Nacionales de Honduras, 2007-2017. Rev Med Hondur. 2018; 86:113-118.

53. Alger J. Fortalecimiento y mejora continua de la Revista Médica Hondureña [Editorial]. Rev Med Hondur. 2019; 87:5.

54. Monge-Nájera J, Ho YS. Honduras publications in the Science Citation Index Expanded: institutions, fields and authors. Rev Biol Trop. 2017; 65:657-668.

55. Decimotercer Congreso Centroamericano y del Caribe de Parasitología y Medicina Tropical. Programa Científico y Resúmenes. Rev Med Hondur. 2017; 85 (Supl. 2): 1-114.

56. García JA, López W, Alger J, Matute ML, Kaminsky RG. Diagnóstico parasitológico en laboratorios públicos y privados en Honduras: ¿Capacidad de respuesta? Rev Med Hondur. 2014;82:148-54.

57. García J, Alger J, Kaminsky R. Actividades de educación continua: Experiencia de la Asociación Hondureña de Parasitología, Tegucigalpa, 2010-2017. Rev Med Hondur. 2018; 86 (Supl. 1): S78.
ABSTRACT. Background: Article commemorating Revista Médica Hondureña 90th anniversary. Objective: To identify articles in parasitology, comment on some publications and document findings. Methodology: Journal issues were consulted at the Honduras Virtual Health Library (www.bvs.hn) to identify articles on different parasitology research themes from 1930 to 2019. Oral presentations in parasitology during national medical congresses were identified in the journal supplements from 2003 to 2019. Results: 234 articles found in parasitology were organized in 12 files, with 73 articles on malaria, 24 in insects/ arachnids, 23 on treatment, 14 on taeniasis/ cysticercosis, 12 on Chagas disease and leishmaniasis, respectively, and others. The lack of research on parasitology was evident. From the 624 oral presentations, 90 (14.0\%) were on parasitology, 40 on malaria and 17 on Chagas disease. Almost no oral presentation was followed by a publication. Discussion: Publications from well conducted research of adequate quality in parasitology are required to further strengthen the health sector and provide viable entries to reduce inequalities. The Honduran Association of Parasitology, founded on June 23, 2000 promotes the development of scientific research together with continuous education in parasitology.

Keywords: Honduras, Parasitology, Publications, Research. 